\title{
Management of patients with macular oedema secondary to central retinal vein occlusion: new findings from SCORE2 and LEAVO studies
}

\author{
Giuseppe Querques $^{1} \cdot$ Riccardo Sacconi $\mathbb{I}^{1} \cdot$ Enrico Borrelli $\mathbb{I}^{1} \cdot$ Francesco Bandello $^{1}$
}

Received: 6 October 2019 / Accepted: 9 October 2019 / Published online: 25 November 2019

(c) The Royal College of Ophthalmologists 2019

Central retinal vein occlusion (CRVO) and its complication, which includes macular oedema (MO), is one of the leading causes of visual impairment [1]. The treatment of MO secondary to CRVO is mainly based on repeated intravitreal antivascular endothelial growth factor (antiVEGF) injections. Three different kinds of anti-VEGF have been used in the clinical practice: the licensed agents (ranibizumab and aflibercept) and an unlicensed low-cost agent (bevacizumab). Bevacizumab is a monoclonal antibody widely used in the treatment of retinal diseases, although its intraocular use is considered off-label. Recently, two different studies, the "Study of Comparative Treatments for Retinal Vein Occlusion 2" (SCORE2) [2] and the "Lucentis, Eylea, Avastin in Vein Occlusion" (LEAVO) trial [3], compared the efficacy of different antiVEGF monotherapies (including Bevacizumab) in the treatment of MO secondary to CRVO. The SCORE2 study reported that bevacizumab was not inferior to aflibercept in the visual acuity outcomes at 6 months [2]. Furthermore, the LEAVO study reported aflibercept to be noninferior compared with ranibizumab in mean changes in vision at 100-week follow-up, but the results of the comparison between bevacizumab and ranibizumab were inconclusive regarding vision outcomes [3].

\section{However, several considerations need to be kept in mind in the interpretation of these data}

In the LEAVO study, the comparison between aflibercept and bevacizumab was not a primary outcome but only a

Giuseppe Querques

giuseppe.querques@hotmail.it

1 Department of Ophthalmology, IRCCS Ospedale San Raffaele, University Vita-Salute, Milan, Italy post-hoc analysis of the study. For this reason, the noninferior results of bevacizumab in comparison to aflibercept, in our opinion, are inconclusive. Taking together this information, the LEAVO study failed to demonstrate a noninferiority of bevacizumab in comparison to ranibizumab and aflibercept.

Another important point regards the safety and the efficacy of the bevacizumab compounding process. Indeed, during the procedures used by pharmacies to compound bevacizumab, the drug could be affected by microbial contamination causing higher levels of infectious endophthalmitis [4]. Furthermore, higher levels of sterile endophthalmitis caused by excess silicone oil residue and particulates were reported $[5,6]$. This could be due to significant higher variability in the protein concentration of bevacizumab compounded from pharmacies in comparison to the drug acquired directly from the manufacturer [7]. The compounding process of bevacizumab causes not only possible microbial contamination and higher protein concentration variability but also a significant lowering and variability of the drug concentration in comparison to the Genentech sample [7]. This variability may have important clinical and ethical implications for ophthalmologists and patients. Despite these findings, to date the majority of clinical trials reported no higher levels of endophthalmitis using bevacizumab in comparison to ranibizumab and aflibercept. However, the bevacizumab used in these series was prepared from compounding pharmacies that may not have been representative of the standard real-life process. Furthermore, no clinical trials were designed to analyse the safety of the drugs, and then the sample size is likely underpowered to detect differences in the endophthalmitis rate.

Great caution should be taken in the comparison between clinical trials and real-life data. For instance, clinical trials have a high rate of treatment adherence and the optimized interval between injections in comparison to the adherence of patients and the number of injections for a year in the clinical practice [2, 8]. In real-life, Italian patients received a lower number of injections in comparison with other 
European countries in the treatment of age-related macular degeneration [8]. This difference reflects the greater visual acuity decline in Italian patients from baseline to year 2 . Real-life studies comparing different anti-VEGF monotherapies with a long term follow-up in CRVO patients should be performed in order to evaluate the outcomes in the clinical practice.

In conclusion, previous studies comparing antiVEGF did not clarify whether bevacizumab is inferior in terms of vision outcome to ranibizumab and aflibercept with a long-term follow-up. Real-life studies are recommended to evaluate the adherence to the therapy, the difference in visual acuity outcomes between anti-VEGF drugs, and the clinical effects of the bevacizumab compounding process.

\section{Compliance with ethical standards}

Conflict of interest GQ is a consultant for Alimera Sciences (Alpharetta, GA, USA), Allergan Inc (Irvine, CA, USA), Amgen (Thousand Oaks, USA), Bayer Shering-Pharma (Berlin, Germany), Heidelberg Engineering Inc (Heidelberg, Germany), KBH (Chengdu, China), LEH Pharma (London, UK), Lumithera (Poulsbo, USA), Novartis (Basel, Switzerland), Sandoz (Berlin, Germany), Sifi (Catania, Italy), Sooft-Fidea (Abano, Italy), Zeiss (Dublin, USA). FB is a consultant for Alcon (Fort Worth, TX, USA), Alimera Sciences (Alpharetta, GA, USA), Allergan Inc (Irvine, CA, USA), Farmila-Thea (Clermont-Ferrand, France), Bayer Shering-Pharma (Berlin, Germany), Bausch And Lomb (Rochester, NY, USA), Genentech (San Francisco, CA, USA), Hoffmann-La-Roche (Basel, Switzerland), Novagali Pharma (Évry, France), Novartis (Basel, Switzerland), Sanofi-Aventis (Paris, France), Thrombogenics (Heverlee, Belgium), Zeiss (Dublin, USA). RS and EB have no conflict of interest.
Publisher's note Springer Nature remains neutral with regard to jurisdictional claims in published maps and institutional affiliations.

\section{References}

1. Sacconi R, Giuffrè C, Corbelli E, Borrelli E, Querques G, Bandello F. Emerging therapies in the management of macular edema: a review. F1000Res 2019;8:F1000. Faculty Rev-1413.

2. Scott IU, VanVeldhuisen PC, Ip MS, Blodi BA, Oden NL, Awh $\mathrm{CC}$, et al. SCORE2 Investigator Group. Effect of bevacizumab vs aflibercept on visual acuity among patients with macular edema due to central retinal vein occlusion: the SCORE2 randomized clinical trial. JAMA. 2017;317:2072-87.

3. Hykin P, Prevost AT, Vasconcelos JC, Murphy C, Kelly J; LEAVO Study Group, et al. Clinical effectiveness of intravitreal therapy with ranibizumab vs aflibercept vs bevacizumab for macular edema secondary to central retinal vein occlusion: a randomized clinical trial. JAMA Ophthalmol. 2019. https://doi.org/10. 1001/jamaophthalmol.2019.3305.

4. Gonzalez S, Rosenfeld PJ, Stewart MW, Brown J, Murphy SP. Avastin doesn't blind people, people blind people. Am J Ophthalmol. 2012;153:196-203, e1.

5. Fielden M, Nelson B, Kherani A. Acute intraocular inflammation following intravitreal injection of bevacizumab: a large cluster of cases. Acta Ophthalmol. 2011;89:e664-e665.

6. Berger AR, Sharma S. Severe intraocular inflammation/endopthalmitis following off-label treatment with intravitreal bevacizumab. Ophthalmology. 2009;1:1-8.

7. Yannuzzi NA, Klufas MA, Quach L, Beatty LM, Kaminsky SM, Crystal RG, et al. Evaluation of compounded bevacizumab prepared for intravitreal injection. JAMA Ophthalmol. 2015;133:32-9.

8. Holz FG, Tadayoni R, Beatty S, Berger A, Cereda MG, Hykin P, et al. Key drivers of visual acuity gains in neovascular age-related macular degeneration in real life: findings from the AURA study. Br J Ophthalmol. 2016;100:1623-8. 